\title{
Millimeter-wave and $\mathrm{THz}$ links under severe weather condition
}

\author{
Tetsuya Kawanishi \\ Faculty of Science and Technology, Waseda University, Japan \\ email: kawanishi@waseda.jp
}

\begin{abstract}
Millimeter-waves and THz-waves can offer high-speed wireless transmission. However, transmission distance would be limited due to atmospheric attenuation and rain attenuation. In addition, mechanical vibration by wind would have impact on link performance, because the wavelengths of carriers are less than a few millimeters. This presentation reviews recent studies on fixed wireless link performance under severe weather condition.
\end{abstract}

Keywords - Millimeter-wave, THz, wind, propagation, atmospheric attenuation.

\section{INTRODUCTION}

High-speed wireless transmission can be realized by using millimeter-waves and THz-waves, because wide frequency bands are available above $100 \mathrm{GHz}$. However, due to large propagation loss in the air, transmission distance would be much shorter than that of conventional radio transmission with low-frequency radio waves. Atmospheric attenuation and additional loss due to rainfall have been studied to design radio transmission and remote sensing systems. In $\mathrm{THz}$ bands, such attenuation much larger than in microwave bands. For example, atmospheric attenuation in $500 \mathrm{GHz}$ band is larger than $50 \mathrm{~dB} / \mathrm{km}$. Impact of wind on radio-wave propagation would be negligible; however, mechanical vibration of antennas causes excess loss in the transmission system. This presentation focuses on wind effect on fixed wireless systems.

\section{THz and millimeter-wave attenuation}

Fig. 1 shows radio-wave attenuation in air $(1-\mathrm{km}$ propagation) calculated by a model defined in Ref. [1]. A blue line shows the attenuation constant for the standard atmosphere defined in Ref. [20, where atmospheric pressure, water vapor pressure, water vapor density and temperature are assumed to be $1013.25 \mathrm{hPa}, 9.9729 \mathrm{hPa}$, $7.5 \mathrm{~g} / \mathrm{m}^{3}$ and $288.15 \mathrm{~K}$. The attenuation constant for dry-air condition is shown with an orange line. For example, the atmospheric attenuation coefficient of $60 \mathrm{GHz}$, where various short-distance radio systems are commercially available, is $14.8 \mathrm{~dB} / \mathrm{km}$ for the standard atmosphere, while that of $300 \mathrm{GHz}$ is $5.6 \mathrm{~dB} / \mathrm{km}$. That implies that $300 \mathrm{GHz}$ bands can offer high-speed wireless links whose transmission distance is longer than in $60 \mathrm{GHz}$. The attenuation constant of $300 \mathrm{GHz}$ is very sensitive to wave vapor density. On the other hand, that of $60 \mathrm{GHz}$ is robust against humidity change, because the large attenuation at
$60 \mathrm{GHz}$ is dominated by absorption by oxygen. Fig. 2 shows attenuation due to rainfall $(20 \mathrm{~mm} / \mathrm{h})$ [3]. The attenuation increases rapidly with the frequency, when the frequency is lower than $100 \mathrm{GHz}$. On the other hand, the attenuation decreases with the frequency in $\mathrm{THz}$ region. Thus, THz links would be robust against rainfalls, where the atmospheric attenuation is much larger than the rainfall attenuation.

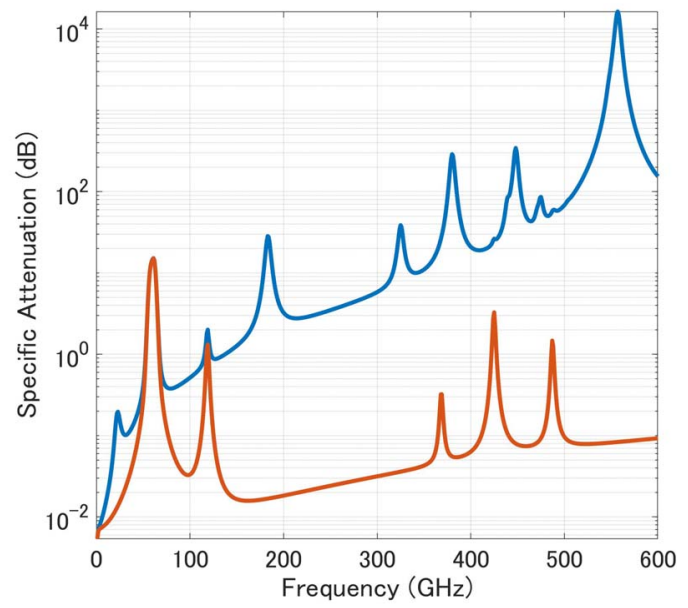

Figure 1. Radio-wave attenuation in air. Blue and orange curves denote attenuation in standard air and dry air.

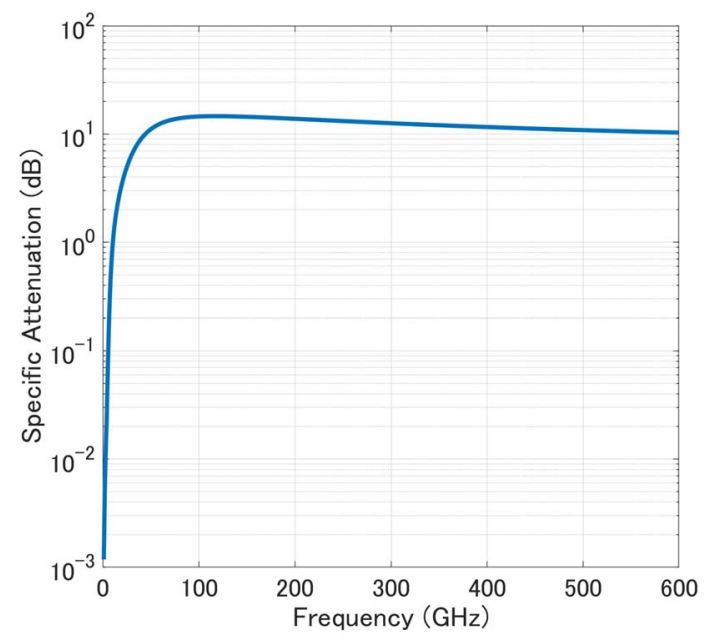

Figure 2. Radio-wave attenuation due to rainfall. 


\section{Wind effect}

Under severe weather condition, the link performance would be degraded by mechanical vibration due to wind, in addition to attenuation or scattering due to rainfalls. A long-term field trial with an E-band millimeter-wave transmission system was performed to measure impact of wind on link performance [4]. Correlation between mechanical vibration and received radio power was measured by using an anemometer and an acceleration sensor were installed on a steel pole. We used a parabolic antenna whose diameter is $350 \mathrm{~mm}$. The thickness and length of the pole were $89 \mathrm{~mm}$ and $5 \mathrm{~m}$, respectively. The average inclination angle of the pole is proportional to the square of the wind speed. The expected inclination of the pole with $20 \mathrm{~m} / \mathrm{s}$ wind is 0.4 degree. For a $10-\mathrm{m}$ pole, the inclination would be 1.6 degree. The half power beam width for the parabolic antenna is $0.9 \mathrm{~dB}$. The $0.4 \mathrm{~dB}$ inclination would cause $2.2 \mathrm{~dB}$ loss in the millimeter-wave link. If we take into account the vibration effect at the receiver side, the loss due to wind would be $4.4 \mathrm{~dB}$ in the worst case.

\section{CONCLUSION}

We discussed $\mathrm{THz}$ link performance under severe weather conditions. In addition to rainfall attenuation, we should take into account mechanical vibration of antennas due to strong wind, in order to design robust $\mathrm{THz}$ wireless transmission system.

\section{ACKNOWLEDGMENT}

The THz-wave transmission study shown in section 2 was performed as a part of Thor (TeraHertz end-to-end wireless systems supporting ultra high data Rate applications) project funded by Horizon 2020, the European Union's Framework Programme for Research and Innovation, under grant agreement No. 814523, and by the Commissioned Research (No. 196) of National Institute of Information and Communications Technology (NICT), Japan..

\section{REFERENCES}

[1] Recommendation ITU-R P.676-11, Attenuation by atmospheric gases and related effects

[2] Recommendation ITU-R P.835-5, Reference Standard Atmospheres

[3] Recommendation ITU-R P.838-3, Specific attenuation model for rain for use in prediction methods.

[4] APT Report on FWS link performance under severe weather conditions APT/AWG/REP-81 